\title{
Quantum quench in two dimensions using the variational Baeriswyl wave function
}

\author{
Balázs Dóra, ${ }^{1, *}$ Masudul Haque, ${ }^{2}$ Frank Pollmann, ${ }^{2}$ and Balázs Hetényi ${ }^{3}$ \\ ${ }^{1}$ Department of Theoretical Physics and BME-MTA Exotic Quantum Phases Research Group, \\ Budapest University of Technology and Economics, 1521 Budapest, Hungary \\ ${ }^{2}$ Max-Planck-Institut für Physik Komplexer Systeme, 01187 Dresden, Germany \\ ${ }^{3}$ Department of Physics, Bilkent University 06800, Ankara, Turkey
}

(Received 30 September 2015; revised manuscript received 3 December 2015; published 15 March 2016)

\begin{abstract}
By combining the Baeriswyl wave function with equilibrium and time-dependent variational principles, we develop a nonequilibrium formalism to study quantum quenches for two-dimensional spinless fermions with nearest-neighbor hopping and repulsion. The variational ground-state energy, the charge-density wave (CDW) order parameter, and the short-time dynamics agree convincingly with the results of numerically exact simulations. We find that, depending on the initial and final interaction strength, the quenched system either exhibits oscillatory behavior or relaxes to a time-independent steady state. The time-averaged expectation value of the CDW order parameter rises sharply when crossing from the steady-state regime to the oscillating regime, indicating that the system, being nonintegrable, shows signs of thermalization with an effective temperature above or below the equilibrium critical temperature, respectively.
\end{abstract}

DOI: 10.1103/PhysRevB.93.115124

\section{INTRODUCTION}

The past decade has witnessed a massive resurgence of interest in the coherent dynamics of quantum many-body systems far from equilibrium [1-3]. This activity has been catalyzed mostly by developments in cold atom experiments $[2,4]$, which allow for long coherence and explicit tracking of the evolution of well-isolated many-body systems in real time. At the same time, there is also a revival of interest in the ultrafast time evolution of solid-state systems. A ubiquitous paradigm in the theoretical study of nonequilibrium dynamics is the so-called quantum quench, where one starts from the ground state of a Hamiltonian, and then instantaneously changes a parameter, so that the system wave function evolves under a different Hamiltonian.

In contrast to the one-dimensional (1D) case, there are only a few exact theoretical methods available to treat real-time evolution in two-dimensional (2D) quantum systems. For example, the density-matrix renormalization-group (DMRG) technique [5-11] is challenging in two dimensions, there are almost no 2D situations that are integrable via the Bethe ansatz, and exact numerical diagonalization is faced with a severely rapid growth of Hilbert space size. There are thus relatively few studies of interaction quenches in two dimensions, e.g., Refs. [12,13] focusing mainly on short-time dynamics and Ref. [14] focusing on time evolution in the absence of interactions.

Variational wave functions are vital in equilibrium condensed-matter physics, e.g., the variational BCS wave function for superconductivity, the Yosida wave function for the Kondo model [15], and the Gutzwiller wave function (GWF) $[16,17]$ for the Hubbard model. The parameters of a well-chosen variational wave function can incorporate the most essential physics of a complex many-body system. In contrast to equilibrium, the nonequilibrium variational principle [18] has been used less extensively in correlated systems.

*dora@eik.bme.hu
The bosonic version of the GWF was often used during the last decade to explore dynamics in the Bose-Hubbard model [19-21]. Recently, computational methods using Monte Carlo evaluation were formulated for the evolution of variational wave functions with large numbers of parameters [22-24]. In addition, the GWF within the Gutzwiller approximation $[16,17]$ was recently used to study the time evolution [25] of the fermionic Hubbard model. This approximation gives the exact solution of the GWF in infinite dimensions [26].

The GWF [16,17] consists of a noninteracting wave function acted on by an operator which projects out states not favored by the interaction (i.e., double occupation for the spinful Hubbard model). A completely opposite variational approach, the Baeriswyl wave function (BWF) $[27,28]$, is based on a fully localized wave function (an eigenfunction of the interaction term) and is acted on by a kinetic-energy projector. The projector has the effect of promoting the hopping of particles in an otherwise completely localized system. In both of these approaches, the strength (coefficient) of the projection operator serves as the variational parameter.

In the present work, we will use the BWF to describe a model of $2 \mathrm{D}$ spinless fermions on the square lattice with nearest-neighbor repulsion at half filling. The reference state is a charge-density wave (CDW) with one of the sublattices exactly filled. An advantage of this approach, as we show, is that it is possible to perform the calculations exactly within the variational manifold, without the requirement of additional approximations. In addition, the wave function is exact at the two extreme limits of infinite and zero interaction. We show that equilibrium properties are well reproduced at all interactions. We use the time-dependent variational principle $[18,29]$ to investigate the dynamics after interaction quenches, and show that the system either relaxes to a steady state or oscillates indefinitely. We map out the "quantum quench phase diagram" according to this criterion and conjecture an interpretation based on the thermalization temperature. The analysis involves characteristics of the CDW order parameter. Density waves have long been a central topic in the study of electronic phases in the solid state [30]. Recent experiments 


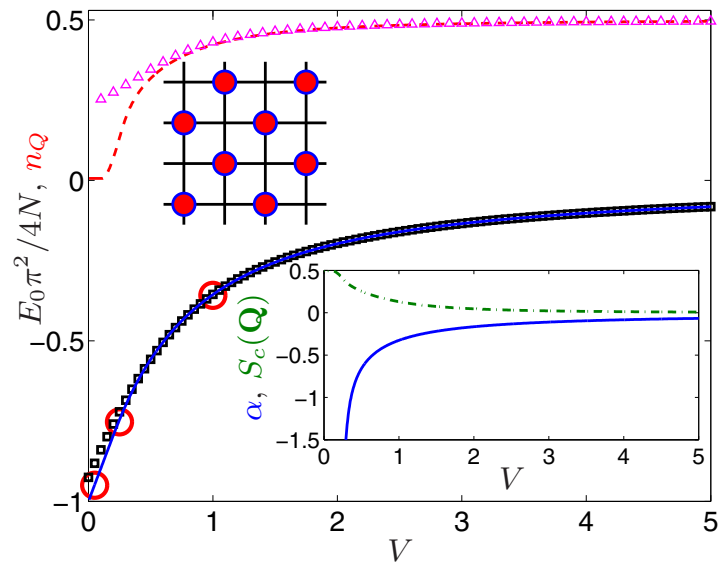

FIG. 1. The variational ground-state energy, normalized by its noninteracting value (blue solid line) and the CDW order parameter (red dashed line), is compared to 2D DMRG data (red circles) and ED on a $4 \times 4$ cluster (black squares, magenta triangles). The lower inset visualizes the equal time structure factor from Eq. (9) for momentum $\mathbf{Q}$ (green dash-dotted line) and the variational parameter $\alpha$ (blue line) as a function of the interaction strength. The upper inset depicts a small segment of the fully polarized CDW state.

have also addressed their nonequilibrium dynamics for CDW [31] as well as of related spin density (antiferromagnetic) patterns [32,33]. The present work studies CDW dynamics for a $2 \mathrm{D}$ interacting system, a topic which at present lies outside the reach of exact methods.

\section{MODEL}

The spinless fermionic Hubbard model is defined on the 2D square lattice as $H=H_{\text {kin }}+H_{\text {int }}$ with

$$
H_{\text {kin }}=-\frac{J}{2} \sum_{\langle\mathbf{n}, \mathbf{m}\rangle} c_{\mathbf{n}}^{+} c_{\mathbf{m}}, \quad H_{\mathrm{int}}=\frac{V}{2} \sum_{\langle\mathbf{n}, \mathbf{m}\rangle} n_{\mathbf{n}} n_{\mathbf{m}},
$$

where $n_{\mathbf{n}}=c_{\mathbf{n}}^{+} c_{\mathbf{n}}$ is the particle density operator at site $\mathbf{n}$, and $c_{\mathbf{n}}$ annihilates a fermion from site $\mathbf{n}$. The sum over $\langle\mathbf{n}, \mathbf{m}\rangle$ runs through all lattice sites and keeps only nearestneighbor pairs. $J$ is the strength of the single-particle hopping integral, used as the unit of energy in the following, thus it is suppressed, and $V>0$ is the strength of the nearest-neighbor Coulomb repulsion. The BWF amounts to starting from the $V \rightarrow \infty$ state and using the kinetic energy as a projection operator [28]:

$$
\left|\Psi_{B}\right\rangle=N_{B}^{-1} \exp \left(\tilde{\alpha} H_{\mathrm{kin}}\right)|\mathrm{CDW}\rangle,
$$

where $|\mathrm{CDW}\rangle$ is the charge ordered ground state $H$ in the atomic limit, where the charges follow a checkerboard pattern on the lattice sites (see Fig. 1), and $N_{B}$ is the overall normalization factor.

The BWF due to its CDW ground state [30] possesses an appealing wave function in momentum space as well. The fully polarized CDW wave function is $|\mathrm{CDW}\rangle=$ $\prod_{\mathbf{k} \in \mathrm{RBZ}} \frac{1}{\sqrt{2}}\left(c_{\mathbf{k}}^{+}+c_{\mathbf{k}-\mathbf{Q}}^{+}\right)|0\rangle$, where $\mathbf{Q}=(\pi / a, \pi / a)$ is the ordering wave vector, $a$ is the lattice constant, the product goes through the reduced (magnetic) Brillouin zone (RBZ), and $|0\rangle$ is the fermionic vacuum. The normalized variational wave function assumes the form

$$
\left|\Psi_{B}\right\rangle=\prod_{\mathbf{k} \in \mathrm{RBZ}} \frac{\exp [\tilde{\alpha} \epsilon(\mathbf{k})] c_{\mathbf{k}}^{+}+\exp [-\tilde{\alpha} \epsilon(\mathbf{k})] c_{\mathbf{k}-\mathbf{Q}}^{+}}{\sqrt{2 \cosh [2 \operatorname{Re} \tilde{\alpha} \epsilon(\mathbf{k})]}}|0\rangle
$$

with $\epsilon(\mathbf{k})=-\cos \left(k_{x} a\right)-\cos \left(k_{y} a\right)$. Here, $\tilde{\alpha}=\alpha+i \eta$ is the complex variational parameter. Its imaginary part becomes relevant when studying the quench problem.

\section{EQUILIBRIUM PROPERTIES}

Due to the structure of the wave function, the problem is more tractable when writing the Hamiltonian in momentum space as

$$
\begin{gathered}
H_{\text {kin }}=\sum_{\mathbf{k}} \epsilon(\mathbf{k}) c_{\mathbf{k}}^{+} c_{\mathbf{k}}, \\
H_{\text {int }}=-\frac{V}{N} \sum_{\mathbf{k}, \mathbf{k}^{\prime}, \mathbf{q}} \epsilon(\mathbf{q}) c_{\mathbf{k}+\mathbf{q}}^{+} c_{\mathbf{k}} c_{\mathbf{k}^{\prime}-\mathbf{q}}^{+} c_{\mathbf{k}^{\prime}}
\end{gathered}
$$

with $N$ the total number of lattice sites. The variational problem is solved by minimizing the ground-state energy with respect to the variational parameter $\tilde{\alpha}$ as $E_{0}=\min _{\tilde{\alpha}}\left\langle\Psi_{B}|H| \Psi_{B}\right\rangle$, and our conventions imply that negative real values of $\tilde{\alpha}$ minimize the above functional, namely, $\alpha<0$ and $\eta=0$. The expectation value of the kinetic and interaction energy is evaluated exactly with the variational wave function as $\left\langle H_{\text {kin }}\right\rangle=\epsilon(\mathbf{0}) I_{2}$ and

$$
\left\langle H_{\mathrm{int}}\right\rangle=-N V \frac{\epsilon(\mathbf{0})}{4}+\frac{V \epsilon(\mathbf{0})}{N} \sum_{i=1,2,3} I_{i}^{2},
$$

and

$$
\begin{aligned}
& I_{1}=\sum_{\mathbf{k}} \frac{\cos [2 \eta \epsilon(\mathbf{k})]}{2 \cosh [2 \alpha \epsilon(\mathbf{k})]}, \\
& I_{2}=\sum_{\mathbf{k}} \frac{\cos \left(k_{x} a\right)}{2} \tanh [2 \alpha \epsilon(\mathbf{k})], \\
& I_{3}=\sum_{\mathbf{k}} \frac{\cos \left(k_{x} a\right) \sin [2 \eta \epsilon(\mathbf{k})]}{2 \cosh [2 \alpha \epsilon(\mathbf{k})]} .
\end{aligned}
$$

The first term in Eq. (6) is the conventional Hartree term, and $I_{1}$ also comes from the Hartree decoupling by taking the anomalous $\left\langle c_{\mathbf{k}}^{+} c_{\mathbf{k}-\mathbf{Q}}\right\rangle \neq 0$ expectation values into account. The Fock terms give rise to $I_{2,3}$, containing normal $\left\langle c_{\mathbf{k}}^{+} c_{\mathbf{k}}\right\rangle$ and anomalous expectation values, respectively. Due to the rotational symmetry of the square lattice, the $x$ and $y$ directions are completely equivalent to each other, hence the $\cos \left(k_{x} a\right)$ factor in $I_{2,3}$, coming from the $\mathbf{q}$ dependence of the interaction, together with the appropriate combinatorial factors, gives the total variational energy. While the interaction term is even in the variational parameters, the kinetic energy is odd in $\alpha$ and independent of $\eta$, therefore their balance determines the optimal variational parameter. Note that we obtain an exact, closed expression for the variational energy, $\left\langle H_{\text {kin }}\right\rangle+\left\langle H_{\text {int }}\right\rangle$ for this model. So far, closed expressions have not been derived based on the GWF for any two-dimensional model. Moreover, the energy expectation values are valid also for hypercubic lattices in arbitrary dimensions $d$, after making the 
replacement $\epsilon(\mathbf{k})=-\sum_{i=1}^{d} \cos \left(k_{i} a\right)$, and the $\mathbf{k}$ sums run over $d$ wave-vector components.

The occupation number of momentum states is $n_{\mathbf{k}}=$ $\left\langle c_{\mathbf{k}}^{+} c_{\mathbf{k}}\right\rangle=1 /\{1+\exp [-4 \alpha \epsilon(\mathbf{k})]\}$. This expression corresponds to the occupation number of a noninteracting system at finite temperature with $T=-1 / 4 \alpha$. The charge density at site $\mathbf{R}$ is

$$
n(\mathbf{R})=\frac{1}{2}+n_{Q} \cos (\mathbf{Q R}), \quad n_{Q}=\frac{I_{1}}{N},
$$

with $n_{Q}$ the CDW order parameter, describing the $\mathbf{Q}$ periodic charge oscillations.

The Qth Fourier component of the equal-time connected structure factor $(\mathrm{SF})$, which measures nonlocal properties and CDW correlations, is

$$
\begin{aligned}
S_{c}(\mathbf{Q}) & =\left\langle\Psi_{B}\left|\rho_{\mathbf{Q}} \rho_{-\mathbf{Q}}\right| \Psi_{B}\right\rangle-N\left|n_{Q}\right|^{2} \\
& =\frac{1}{2}-\frac{1}{2 N} \sum_{\mathbf{k}} \frac{\cos ^{2}[2 \eta \epsilon(\mathbf{k})]}{\cosh ^{2}[2 \alpha \epsilon(\mathbf{k})]},
\end{aligned}
$$

where $\rho_{\mathbf{Q}}=\frac{1}{\sqrt{N}} \sum_{\mathbf{k}} c_{\mathbf{k}}^{+} c_{\mathbf{k}-\mathbf{Q}}$, and gives zero in the fully polarized CDW state and $1 / 2$ in the absence of CDW correlations. Therefore, the value of the SF is related to the amount of CDW order in the system. It is time independent in equilibrium, shown in Fig. 1, and reflects the behavior of $n_{Q}$.

The variational wave function is exact in two opposite limits, $V=0(\alpha=-\infty)$ and $V=\infty(\alpha=0)$. In the former case, the ground-state energy is $E_{0}=-4 N / \pi^{2}$, while for the latter the kinetic energy is suppressed by the CDW and the interaction energy reaches its minimum, therefore $E_{0}=0$. In between these two extrema, the ground-state energy, the CDW order parameter $n_{Q}$, and the optimal variational parameter are shown in Fig. 1, together with numerical data using 2D DMRG and exact diagonalization (ED) on a $4 \times 4$ cluster. To detect $\mathrm{CDW}$ ordering, we compute density-density correlations as $n_{Q}=\sqrt{\sum_{\mathbf{n}, \mathbf{m}}\left\langle n_{\mathbf{n}} n_{\mathbf{m}}\right\rangle \exp [i \mathbf{Q}(\mathbf{n}+\mathbf{m})]} / N$ using ED. The 2D DMRG data are obtained by extrapolating the energies for infinitely long cylinders with circumferences $L=6,8$, and 10 to the thermodynamic limit. The agreement between the variational and numerically obtained energies and CDW order parameter is excellent. The ED matches the DMRG data for larger $V$, indicating short correlation length, such that the relatively small system size does not influence physical observables.

The system is always in a CDW state, except for $\alpha \rightarrow-\infty$, corresponding to the noninteracting limit $V=0$. This is in agreement with what is expected for the spinless Hubbard model on the square lattice $[34,35]$. The CDW phase appears because of the square shaped Fermi surface at half filling, in the case of perfect nesting [30], and also due to the log-divergent density of states upon approaching half filling as $\sim \ln (1 / \omega)$.

\section{QUANTUM QUENCH}

We now turn to the investigation of the quantum quench, when an initial $V_{i}$ is changed suddenly to $V_{f}$ at $t=0$. The time-dependent wave function is of Baeriswyl form, $\left|\Psi_{B}(t)\right\rangle$ as well, and the quench amounts to allowing for time-dependent variational parameters $\alpha(t)$ and $\eta(t)$ in Eq. (3).

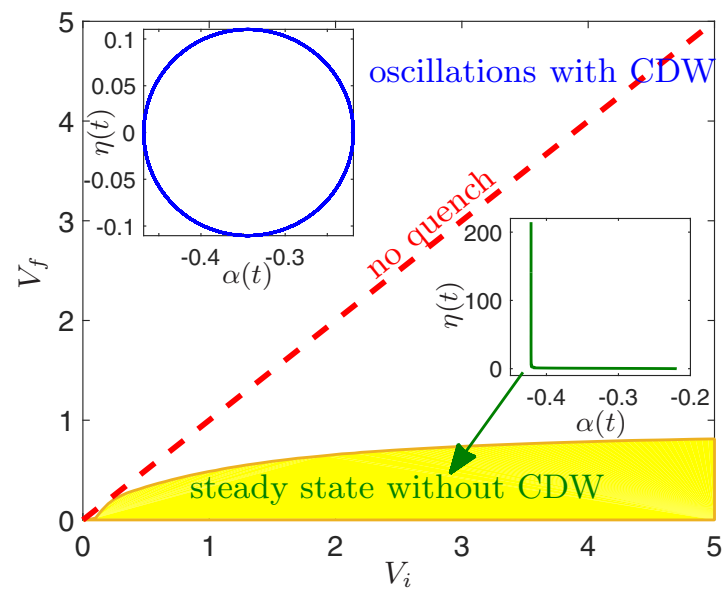

FIG. 2. The quantum quench phase diagram for $V_{i}$ and $V_{f}$ is shown. For small $V_{f}$, a time-independent steady state is reached which could be interpreted as an indicator thermalization at an effective temperature higher than the equilibrium CDW transition temperature with $\eta(t \rightarrow \infty) \sim t$ and $\alpha(t)$ saturating to a finite value, as depicted in the lower inset for $V_{i}=1.5, V_{f}=0.25$ and $0<t<200$. For sizable quenches, on the other hand, the system periodically returns to its initial state, visualized in the upper inset for $V_{i}=1.5, V_{f}=1$. For $V_{i}=\infty$, the transition from the steady state to oscillating behavior occurs at $V_{f} \approx 0.97$.

Their time dependence follows from the time-dependent variational principle, which requires the minimization of the real Lagrangian, defined as $[18,29]$

$$
L(t)=-\operatorname{Im}\left(\left\langle\Psi_{B} \mid \partial_{t} \Psi_{B}\right\rangle\right)-\left\langle\Psi_{B}|H| \Psi_{B}\right\rangle,
$$

with respect to the time-dependent variational parameters. Using $L(t)=\left\langle H_{\text {kin }}\right\rangle\left(\partial_{t} \eta-1\right)-\left\langle H_{\text {int }}\right\rangle$ with the expectation values taken from Eq. (6) with the time-dependent variational parameters, we finally arrive to the Euler-Lagrange equations, determining the quench dynamics as

$$
\partial_{t} \alpha=\frac{\frac{\partial\left\langle H_{\text {int }}\right\rangle}{\partial \eta}}{\frac{\partial\left\langle H_{\text {kin }}\right\rangle}{\partial \alpha}}, \quad \partial_{t} \eta=1+\frac{\frac{\partial\left\langle H_{\text {int }}\right\rangle}{\partial \alpha}}{\frac{\partial\left\langle H_{\text {kin }}\right\rangle}{\partial \alpha}},
$$

supplemented with the initial conditions $\alpha(0)=\alpha_{i}$, originating from the equilibrium configuration for $V_{i}$, and $\eta(0)=0$. From these, the total energy is conserved after the quench, as expected.

From Eq. (11), the two limiting cases are recovered. First, when no quench was performed, $\alpha(t)=\alpha_{i}$ and $\eta(t)=0$. Second, in the case of quenching from $V_{i}=\infty$ to $V_{f}=0$, the $\alpha_{i}=0$ initial condition fixes $\alpha(t)=0$, while $\eta(t)=t$. For general values of initial and final interactions, one needs to integrate these coupled differential equations numerically (see the Appendix). The obtained phase diagram is shown in Fig. 2. Equation (1) in two dimensions is nonintegrable, therefore it is expected to thermalize after a sudden quench, and we argue that our simple variational scheme is able to capture some of its physics.

The time dependence of the CDW order parameter is calculated from Eq. (8) after inserting the time-dependent variational parameters into $I_{1}$. The short-time behavior from 

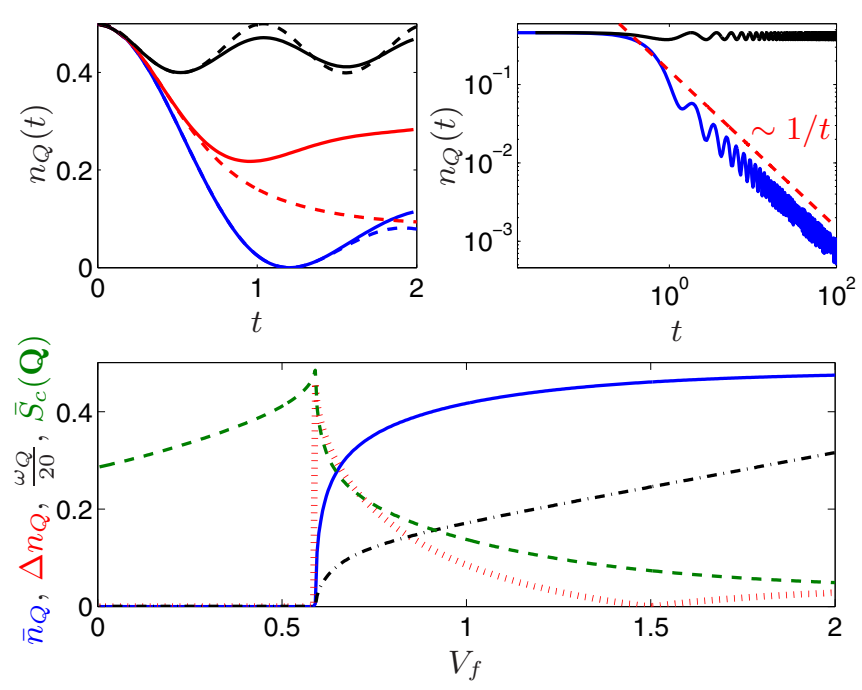

FIG. 3. Top left: The short-time decay of the CDW order parameter from time-dependent 2D DMRG for an infinite cylinder of circumference $L=8$ (solid lines) and variational calculation (dashed lines) for $V_{i}=\infty$ and $V_{f}=0$ (blue), 1 (red) and 2 (black). Top right: The typical behavior of the CDW order parameter is plotted in the steady-state region (blue line), vanishing as $1 / t$, for $V_{i}=1.5$ and $V_{f}=0.25$ and in the oscillatory region (black line) for $V_{i}=1.5$ and $V_{f}=1$. Bottom: The time average value of the CDW order parameter (blue solid line), the amplitude (red dotted line), and the frequency (black dash-dotted line) of oscillations, together with the structure factor for momentum $\mathbf{Q}$ (green dashed line) are shown for $V_{i}=1.5$.

the BWF agrees well with that from 2D time-dependent DMRG [36] obtained using the algorithm introduced in Ref. [11], as shown in Fig. 3. The $V_{i}=\infty \rightarrow V_{f}=0$ quench is exact within the variational framework, which also allows us to check the temporal validity of the numerics.

For longer times, the variational solution either oscillates around its time average or reaches a steady state after the quench (see the Appendix), which is the typical behavior [37] in similar approaches. For quenches with small $V_{f}$, a time-independent steady state is reached with $\eta(t)$ increasing linearly with time and $\alpha(t)$ saturating to a fixed value. This can be interpreted through the fact that a stronger quench pumps more energy into the system compared to the final ground state. As a result, it eventually thermalizes to a higher effective temperature than the equilibrium CDW transition temperature, thus $\mathrm{CDW}$ is absent. The CDW order parameter decays to zero since due to $\eta(t) \sim t$ the numerator in $I_{1}$ oscillates fast and kills the integral with increasing $t$. In particular, for arbitrary spatial dimension $d$, a $n_{Q}(t) \sim t^{-d / 2}$ decay is found, in accordance with special quenches of the 1D Heisenberg chain [38]. A similar decay is found for the antiferromagnetic order of the 2D spinful Hubbard model [14], quenched to a noninteracting system. While in one dimension the CDW order parameter oscillates and also changes sign after the quench [14,38], it only oscillates around its envelope function in two dimensions but does not change sign, as shown in Fig. 3. This is supported by the exact result when quenching from $V_{i}=\infty$ to $V_{f}=0$, $n_{Q}(t)=J_{0}^{d}(2 t) / 2$, valid in arbitrary dimension $d$, and $J_{0}(x)$ is the zeroth Bessel function of the first kind, in agreement with our numerical findings.
For larger $V_{f}$, on the other hand, an oscillating solution is found for both $\alpha(t)$ and $\eta(t)$, and the system periodically returns to its initial state, and seemingly does to reach a time-independent steady state, illustrated in Fig. 2. Within our approach, we interpret this as an indicator of thermalization to a thermal state with lower effective temperature than the equilibrium CDW transition temperature, hence the resulting state possesses a finite CDW order. The oscillations are common in variational calculations [37], but the time-averaged values correspond to that in the steady state. Throughout the time evolution, $\alpha(t)$ stays negative (see the Appendix).

In order to further characterize the CDW phase, we consider the time average of the order parameter as $\bar{n}_{Q}=$ $\lim _{t \rightarrow \infty} \frac{1}{t} \int_{0}^{t} n_{Q}\left(t^{\prime}\right) d t^{\prime}$, together with the amplitude $\Delta n_{Q}$ and frequency $\omega_{Q}$ of the oscillations of $n_{Q}(t)$ in the oscillating regime, shown in Fig. 3 for the representative case of $V_{i}=1.5$. Here, $\Delta n_{Q}=\lim _{t \rightarrow \infty}\left\{\max \left[n_{Q}(t)\right]-\min \left[n_{Q}(t)\right]\right\}$ in the oscillating regime and $\omega_{Q}$ is the basic harmonics of the oscillations in $n_{Q}(t)$, evaluated from Fourier analysis. Due to the nonintegrability of the model, the system is expected to thermalize [2]. For small $V_{f}$, the CDW gap and transition temperature is small and the effective temperature the system reaches is above the equilibrium CDW transition temperature, therefore the CDW order is absent in this case, as evidenced in Fig. 3. For larger final interaction, however, the corresponding equilibrium transition temperature increases and becomes equal to the effective temperature of the thermalized system, which marks the sharp rise of $\bar{n}_{Q}$. From that point on, with increasing $V_{f}$, the system's effective temperature is always smaller than the equilibrium transition temperature, therefore the system exhibits long-range CDW order, mimicking a thermalized state. This scenario is further corroborated by focusing on other characteristics of the order, such as the amplitude and frequency of the oscillations in the oscillating regime.

The equal time Qth structure factor after the quench is obtained by inserting the time-dependent variational parameters in Eq. (9). Its long-time average $\bar{S}_{c}(\mathbf{Q})$ is plotted in Fig. 3. At the critical $V_{f}$, where the transition occurs, the variational wave function is almost metallic and contains enhanced CDW correlations away from this point. However, only strong interaction can profit from these correlations and drive the dynamical CDW transition. The effective temperature after the quench is expected to increase monotonically with $\left|V_{f}-V_{i}\right|$, while the equilibrium CDW gap grows with $V_{f}$. When these two energy scales become comparable, the dynamical phase transition occurs.

\section{COMPARISON TO GUTZWILLER WAVE FUNCTION}

The GWF was shown [39] to be conducting for finite interaction strength in finite dimension for any model, a result corroborated by the exact results for the Hubbard model in one dimension [40]. The GWF only produces a metal-insulator transition when solved within the Gutzwiller approximation $[16,17]$, which is exact in the infinite dimensional case [26], but is a drastic approximation in finite dimensions. In this approximation the Pauli principle is taken into account 
combinatorically, without explicit reference to the antisymmetry of the wave function. In contrast, while an exact solution to the BWF is not available, it can be shown [41] that it produces an insulating state for any interaction strength in finite dimensions, a result in quantitative agreement with our numerics. We stress that the equilibrium properties of a model which is insulating at finite interaction strength are not reproduced by the exact solution of the GWF. Given that the model we study here is insulating in two dimensions, it is reasonable to expect that the BWF captures its properties better. Additionally, the GWF, when solved within a timedependent generalization of the Gutzwiller approximation, never reaches a time-independent steady state [25] out of equilibrium, unlike the BWF. The BWF thus is superior to GWF for the present problem, as it not only accounts for the equilibrium properties of our model but has predictive power for the out of equilibrium dynamics as well.

\section{CONCLUSION}

We have studied quantum quenches for 2D spinless fermions on the square lattice with nearest-neighbor hopping and repulsion using the variational BWF, which are unaccessible by exact methods. Depending on the initial and final interaction strength, the system either reaches a time-independent steady state or oscillates periodically in time. We argue by investigating the characteristics of the CDW order parameter and the equal time structure factor that the former and latter behave similarly to what is expected from a thermal state with effective temperature larger and smaller than the equilibrium CDW transition temperature, respectively.

Our work opens up a number of interesting questions worth pursuing in the future, such as considering bosons instead of fermions, the effect of disorder, the influence of imperfect nesting, incorporating the spin degree of freedom, as well as the improvement of the variational method by introducing variational parameters for each $\mathbf{k}$ mode.

\section{ACKNOWLEDGMENTS}

We thank Johannes Motruk for sharing two-dimensional density-matrix renormalization-group data for the equilibrium case. B.D. was supported by the Hungarian Scientific Research Funds Grants No. K101244, No. K105149, and No. K108676 and by the Bolyai Program of the Hungarian Academy of
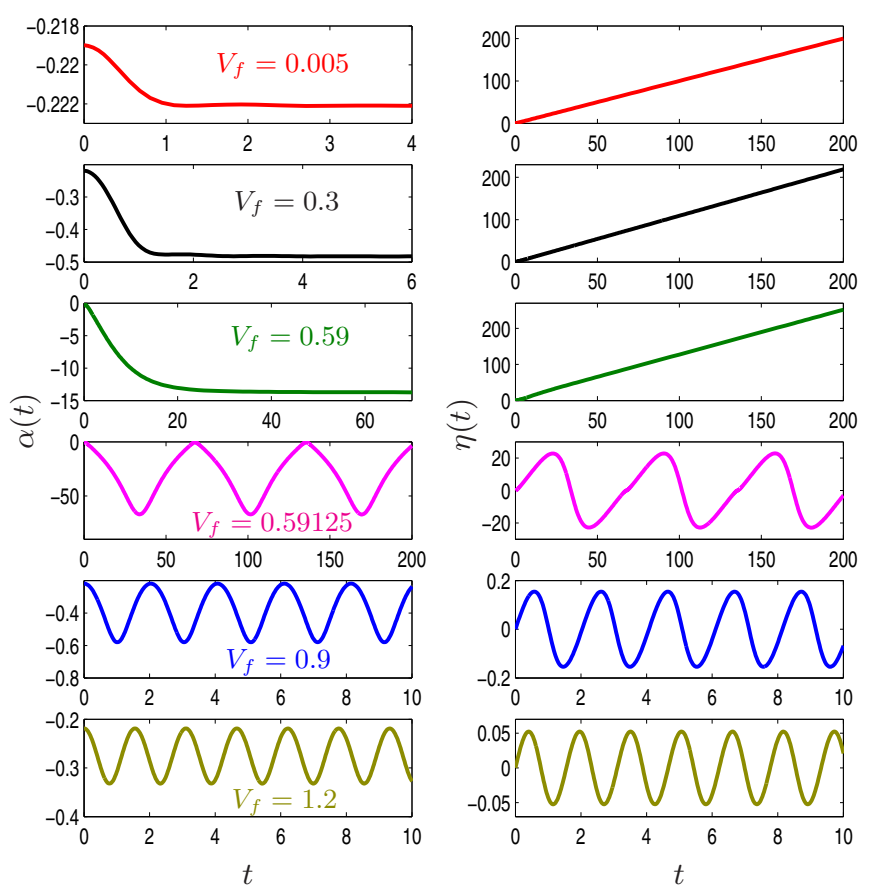

FIG. 4. The time dependence of the variational parameters is plotted for $V_{i}=1.5$ and several final $V_{f}$. The critical interaction strength separating the steady state and oscillating regimes is around $V_{f} \approx 0.591$. Note the different horizontal and vertical scales.

Sciences. B.H. was supported by the Turkish national agency for basic research (TUBITAK Grant No. 133F344).

\section{APPENDIX: TIME DEPENDENCE OF THE VARIATIONAL PARAMETERS}

The numerical solution of Eqs. (11) in the main text yields the time-dependent variational parameters, the behavior of which is plotted in Fig. 4. In the steady-state regime, the $\eta(t)$ keeps on increasing linearly with time, while $\alpha(t)$ reaches a time-independent steady-state value. However, the relaxation time required to reach this increases with increasing $V_{f}$. At a critical final interaction strength, the solution changes abruptly and both parameters exhibit periodic oscillations. The oscillation frequency increases sharply with $V_{f}$, and contains higher harmonics as well close to the critical interaction strength. With increasing $V_{f}$, however, a single frequency describes more and more reliably the data.
[1] J. Dziarmaga, Adv. Phys. 59, 1063 (2010).

[2] A. Polkovnikov, K. Sengupta, A. Silva, and M. Vengalattore, Rev. Mod. Phys. 83, 863 (2011).

[3] J. Eisert, M. Friesdorf, and C. Gogolin, Nat. Phys. 11, 124 (2015).

[4] I. Bloch, J. Dalibard, and W. Zwerger, Rev. Mod. Phys. 80, 885 (2008).

[5] S. R. White, Phys. Rev. Lett. 69, 2863 (1992).
[6] J. A. Kjäll, M. P. Zaletel, R. S. K. Mong, J. H. Bardarson, and F. Pollmann, Phys. Rev. B 87, 235106 (2013).

[7] U. Schollwöck, Ann. Phys. 326, 96 (2011).

[8] S. R. Manmana, A. Muramatsu, and R. M. Noack, AIP Conf. Proc. 789, 269 (2005).

[9] P. R. Zangara, A. D. Dente, E. J. Torres-Herrera, H. M. Pastawski, A. Iucci, and L. F. Santos, Phys. Rev. E 88, 032913 (2013). 
[10] A. Daley, C. Kollath, U. Schollwöck, and G. Vidal, J. Stat. Mech.: Theory Exp. (2004) P04005.

[11] M. P. Zaletel, R. S. K. Mong, C. Karrasch, J. E. Moore, and F. Pollmann, Phys. Rev. B 91, 165112 (2015).

[12] S. A. Hamerla and G. S. Uhrig, Phys. Rev. B 89, 104301 (2014).

[13] J. Hauschild, F. Pollmann, and F. Heidrich-Meisner, Phys. Rev. A 92, 053629 (2015).

[14] F. Goth and F. F. Assaad, Phys. Rev. B 85, 085129 (2012).

[15] K. Yosida, Phys. Rev. 147, 223 (1966).

[16] M. C. Gutzwiller, Phys. Rev. Lett. 10, 159 (1963).

[17] M. C. Gutzwiller, Phys. Rev. 137, A1726 (1965).

[18] P. Kramer and M. Saraceno, Geometry of the Time-Dependent Variational Principle in Quantum Mechanics (Springer-Verlag, Berlin, 1981).

[19] D. S. Rokhsar and B. G. Kotliar, Phys. Rev. B 44, 10328 (1991).

[20] D. Jaksch, V. Venturi, J. I. Cirac, C. J. Williams, and P. Zoller, Phys. Rev. Lett. 89, 040402 (2002).

[21] M. Jreissaty, J. Carrasquilla, F. A. Wolf, and M. Rigol, Phys. Rev. A 84, 043610 (2011).

[22] G. Carleo, F. Becca, M. Schiró, and M. Fabrizio, Sci. Rep. 2, 243 (2012).

[23] G. Carleo, F. Becca, L. Sanchez-Palencia, S. Sorella, and M. Fabrizio, Phys. Rev. A 89, 031602 (2014).

[24] K. Ido, T. Ohgoe, and M. Imada, Phys. Rev. B 92, 245106 (2015).

[25] M. Schiró and M. Fabrizio, Phys. Rev. Lett. 105, 076401 (2010).

[26] W. Metzner and D. Vollhardt, Phys. Rev. Lett. 62, 324 (1989).
[27] D. Baeriswyl, in Nonlinearity in Condensed Matter, edited by A. R. Bishop, D. K. Campbell, D. Kumar, and S. E. Trullinger (Springer-Verlag, New York, 1986).

[28] B. Valenzuela, S. Fratini, and D. Baeriswyl, Phys. Rev. B 68, 045112 (2003).

[29] J. Bünemann, S. Wasner, E. v. Oelsen, and G. Seibold, Phil. Mag. 95, 550 (2014).

[30] G. Grüner, Density Waves in Solids (Addison-Wesley, Reading, 1994).

[31] S. Trotzky, Y.-A. Chen, A. Flesch, I. P. McCulloch, U. Schollwöck, J. Eisert, and I. Bloch, Nat. Phys. 8, 325 (2012).

[32] P. J. Lee, M. Anderlini, B. L. Brown, J. Sebby-Strabley, W. D. Phillips, and J. V. Porto, Phys. Rev. Lett. 99, 020402 (2007).

[33] S. Trotzky, P. Cheinet, S. Fölling, M. Feld, U. Schnorrberger, A. M. Rey, A. Polkovnikov, E. A. Demler, M. D. Lukin, and I. Bloch, Science 319, 295 (2008).

[34] M. S. Foster and A. W. W. Ludwig, Phys. Rev. B 77, 165108 (2008).

[35] J. de Woul and E. Langmann, J. Stat. Phys. 139, 1033 (2010).

[36] For longer times, DMRG becomes unreliable due to entanglement growth and the finite circumference of the infinite cylinder.

[37] B. Sciolla and G. Biroli, J. Stat. Mech.: Theory Exp. (2011) P11003.

[38] P. Barmettler, M. Punk, V. Gritsev, E. Demler, and E. Altman, New J. Phys. 12, 055017 (2010).

[39] A. J. Millis and S. N. Coppersmith, Phys. Rev. B 43, 13770 (1991).

[40] W. Metzner and D. Vollhardt, Phys. Rev. B 37, 7382 (1988).

[41] M. Dzierzawa, D. Baeriswyl, and L. M. Martelo, Helv. Phys. Acta 70, 124 (1997). 